\title{
ANNIHILATORS AND THE CS-CONDITION
}

\author{
by W. K. NICHOLSON and M. F. YOUSIF
}

(Received 8 October, 1996)

\begin{abstract}
It is proved that if every cyclic right $R$-module is torsionless and $R$ is a left CS-ring then $R$ is semiperfect left continuous with $\operatorname{soc}\left(R_{R}\right)$ essential in ${ }_{R} R$. As a consequence every right cogenerator, left CS-ring $R$ is shown to be right pseudo-Frobenius and left continuous, and an example is given to show that $R$ need not be left selfinjective. It is also proved that if $R$ is a left CS-ring and every cyclic right $R$-module embeds in a free module, then $R$ is quasi-Frobenius if and only if $J(R) \subseteq Z\left(R_{R}\right)$.
\end{abstract}

1. Introduction. Right CS-rings with certain cogenerating (annihilator) conditions were considered by Gomez-Pardo and Guil Asensio in [10] and [11]. For example they show that if $R$ is a right CS-ring and every cyclic (finitely generated) right $R$-module embeds in a free module then $R$ is right artinian (quasi-Frobenius). They also prove that if $R$ is a right cogenerator right CS-ring then $R$ is right pseudo-Frobenius.

In this paper we consider these same classes of rings but with the left CS-condition rather than the right CS-condition. We show that if $R$ is a left CS-ring and every cyclic right $R$-module is torsionless, then $R$ is a semiperfect left continuous ring with $\operatorname{soc}\left(R_{R}\right) \subseteq{ }^{e s s} R$. We use this result to show that if $R$ is a right cogenerator left CS-ring then $R$ is a right pseudo-Frobenius, left continuous ring. An example of Dischinger and Müller [5] shows that the ring $R$ need not be left selfinjective. We also prove that if $R$ is a left CS-ring and every cyclic right $R$-module embeds in a free module, then $R$ is quasi-Frobenius if and only if $J(R) \subseteq Z\left(R_{R}\right)$.

Throughout this paper every ring $R$ is associative with unity and all modules are unitary. If $M_{R}$ is a right $R$-module we write $J(M), Z(M), \operatorname{soc}(M)$ and $E(M)$ for the Jacobson radical, the singular submodule, the socle and the injective hull of $M$, respectively. We denote the direct sum of $k$ copies of $M$ by $M^{(k)}$, and the notation $K \subseteq{ }^{e s s} M$ means that $K$ is an essential submodule of $M$.

We frequently refer to the following conditions on a module $M_{R}$ :

The $C_{1}$-condition (or the $C S$-condition): Every submodule of $M$ is essential in a direct summand of $M$.

The $C_{2}$-condition: Every submodule of $M$ that is isomorphic to a summand of $M$ is itself a summand of $M$.

The module $M_{R}$ is called continuous if $M$ satisfies both the $\mathrm{C}_{1}$ - and $\mathrm{C}_{2}$-conditions. The ring $R$ is called right CS (right continuous) if $R_{R}$ is a CS-module (a continuous module).

The left (respectively right) annihilator of a subset $X$ of a ring $R$ is denoted $l(X)$ (respectively $r(X)$ ). The ring $R$ is called right Kasch if every simple right $R$-module embeds in $R$ (equivalently if $l(T) \neq 0$ for every maximal right ideal $T$ of $R$ ). A right $R$-module $M$ is

Glasgow Math. J. 40 (1998) 213-222. 
called torsionless if $M$ is embedded in a direct product of copies of $R$. We often use the fact that, if $T$ is a right ideal of $R$, then $R / T$ is torsionless as a right $R$-module if and only if $r l(T)=T$. A ring $R$ is called right cogenerator if every right $R$-module is torsionless, and $R$ is called a right $P F$-ring (pseudo-Frobenius ring) if it is right cogenerator and right selfinjective (equivalently if it is semiperfect, right selfinjective and $\left.\operatorname{soc}\left(R_{R}\right) \subseteq{ }^{\text {ess }} R_{R}\right)$. In [15] $R$ is called a right GPF-ring (generalized pseudo-Frobenius ring) if $R$ is semiperfect, right P-injective and $\operatorname{soc}\left(R_{R}\right) \subseteq{ }^{e s s} R_{R}$. Here $R$ is called right $P$-injective (principally injective) if every $R$-homomorphism from a principal right ideal of $R$ into $R$ is given by left multiplication.

2. Annihilators and the CS-condition. We begin with a basic fact about annihilators of right ideals which will be used several times.

Lemma 2.1. Let $\mathcal{C}$ be a class of right ideals of $R$ with the property that $T \in \mathcal{C}$ implies $b T \in \mathcal{C}$ for all $b \in R$. The following conditions are equivalent:

(1) $r l(T)=T$ for all $T \in \mathcal{C}$.

(2) $r[l(T) \cap R b]=T+r(b)$ for all $T \in \mathcal{C}$ and all $b \in R$.

Proof. Clearly (2) $\Rightarrow(1)$. Given (1), observe that $T+r(b) \subseteq r[l(T) \cap R b]$ always holds. If $x \in r[l(T) \cap R b]$, then $l(b T) \subseteq l(b x)$ (indeed, if $y \in l(b T)$ then $y b T=0$, so $y b \in l(T) \cap R b$, whence $y b x=0)$. But then $b x \in r l(b x) \subseteq r l(b T)=b T$ by hypothesis. If $b x=b t$ for $t \in T$, then $(x-t) \in r(b)$ so $x \in T+r(b)$, as required.

RemarK. The class $\mathcal{C}$ in Lemma 2.1 could be any of the following classes of right ideals: all; finitely generated; principal; semisimple; minimal or zero; small; singular.

Lemma 2.2. Suppose that $r(T)=T$ for all right ideals $T$ of $R$. If every complement left ideal of $R$ is principal, then $R$ is semiperfect.

Proof. Let $T$ be a right ideal, and let $L$ be a left ideal maximal with respect to $l(T) \cap L=0$. By hypothesis, $L=R b, b \in R$, so $T+r(b)=R$ by Lemma 2.1. It suffices (see [14], Theorem 11.1.5) to show that $r(b)$ is minimal with respect to this property (that is, $T$ has an additive complement in $R$ ). But if $R=T+C$ with $C \subseteq r(b)$, then $L=R b \subseteq \operatorname{lr}(b) \subseteq l(C)$. Since $l(T) \cap l(C)=0$, the choice of $L$ gives $L=l(C)$, so $C=r l(C)=r(L)=r(b)$, as required.

Lemma 2.3. Suppose that $R$ is a right Kasch, left CS-ring. Then $R$ is left continuous with $\operatorname{soc}\left(R_{R}\right) \subseteq{ }_{R}^{\text {ess }} R$.

Proof. Every right Kasch ring satisfies the left $C_{2}$-condition (see [21], Lemma 1.15), so $R$ is left continuous. By the left CS-condition, let $\operatorname{soc}\left(R_{R}\right) \subseteq \subseteq^{e s s} R e$ for $e^{2}=e \in R$, so that $1-e \in r\left[\operatorname{soc}\left(R_{R}\right)\right]$. But $r\left[\operatorname{soc}\left(R_{R}\right)\right]=J(R)$ because $R$ is right Kasch, so $1-e \in J(R)$. This means that $e=1$, and so $\operatorname{soc}\left(R_{R}\right) \subseteq{ }^{e s s} R$.

Proposition 2.4. Suppose that $R$ is a left $C S$-ring for which every cyclic right $R$-module is torsionless. Then $R$ is a semiperfect, left continuous ring with $\operatorname{soc}\left(R_{R}\right) \subseteq{ }_{R}^{\text {ess }} R$. In particular, $R$ is left finite dimensional. 
Proof. We have $r l(T)=T$ for every right ideal $T$ because $R / T$ is torsionless. In particular, $R$ is right Kasch and so is left continuous with $\operatorname{soc}\left(R_{R}\right) \subseteq \subseteq^{\text {ess }}{ }_{R} R$ by Lemma 2.3. Furthermore, the left CS-condition shows that every complement left ideal is a summand, and so is principal. Thus $R$ is semiperfect by Lemma 2.2, so write $R=R e_{1} \oplus \cdots \oplus R e_{n}$ where each $e_{i}$ is a local idempotent. Then each $R e_{i}$ is a CS-module and so is uniform. Thus $R$ is left finite dimensional.

It was proved by Gomez Pardo and Guil Asensio [11] that, for a right cogenerator ring $R$, right CS implies right selfinjective. In other words, $R$ is a right PF-ring (that is $R$ is right cogenerator, right selfinjective) if and only if $R$ is a right cogenerator, right CS-ring. This theorem extends all the known results on the subject. On the other hand, it is well-known [7] that a ring $R$ is a left and right PF-ring if and only if $R$ is a right cogenerator, left selfinjective ring. So it is natural to ask whether the result of Gomez Pardo and Guil Asensio can be obtained if we replace the right CS-condition by the left CS-condition. In fact we have

Proposition 2.5. Let $R$ be a right cogenerator ring.

(1) If $R$ is left $C S$ then $R$ is left continuous and right selfinjective (and so is right $P F$ ).

(2) If $R \oplus R$ is $C S$ as a left $R$-module then $R$ is left and right $P F$.

Proof. (1) $R$ is a semiperfect, left continuous ring by Proposition 2.4. In particular $R$ has a finite number of isomorphism classes of simple right (and left) $R$-modules. Since $R$ is a right cogenerator, $R$ is right selfinjective by [7, Proposition 24.9], and hence is a right PF-ring.

(2) $R$ is left continuous by (1), so is left selfinjective by [21, Proposition 1.21]. Hence $R$ is a left PF-ring; it is right PF by (1).

REMARK. A right cogenerator, left CS-ring need not be left selfinjective. In fact, an example of Dischinger and Müller [5] shows the existence of a local, left continuous right PF-ring which is not left selfinjective.

Before proceeding, we record a fact about embeddings which will be used later. The proof is straightforward.

Lemma 2.6. If a uniform module $U$ can be embedded in $M_{1} \oplus \cdots \oplus M_{n}$ where each $M_{i} \neq 0$, then $U$ embeds in $M_{k}$ for some $k=1, \cdots, n$.

In order to strengthen Proposition 2.5, we need the following well known lemma. Recall that a module $M$ is called finitely embedded (finitely cogenerated) if $M$ has a finitely generated essential socle.

Lемма 2.7. Every finitely embedded torsionless right $R$-module $M$ embeds in a free module $R^{n}$ of finite rank $n$.

Proof. See for example [8, Corollary 3.1.B] or [1, Propositions 10.2 and 10.7].

THEOREM 2.8. The following conditions are equivalent for a left CS-ring $R$.

(1) $R$ is a right $P F$-ring.

(2) $J(R) \subseteq Z\left(R_{R}\right)$ and every 2-generated right $R$-module is torsionless. 
Proof. (1) $\Rightarrow(2)$. Every right $\mathrm{PF}$-ring is a right selfinjective, right cogenerator ring.

$(2) \Rightarrow(1)$. By Proposition 2.4, $R$ is a semiperfect, left continuous ring with $\operatorname{soc}\left(R_{R}\right) \subseteq{ }^{e s s}{ }_{R} R$. The fact that $r l(T)=T$ for all right ideals $T$ implies that $R$ is left P-injective, so $\operatorname{soc}\left({ }_{R} R\right) \subseteq \operatorname{soc}\left(R_{R}\right)$ by Theorem 1.14 in [16]. Thus, using (2),

$$
\operatorname{soc}\left({ }_{R} R\right) \subseteq \operatorname{soc}\left(R_{R}\right) \subseteq r\left[Z\left(R_{R}\right)\right] \subseteq r[J(R)]=\operatorname{soc}\left({ }_{R} R\right)
$$

and it follows that $\operatorname{soc}\left({ }_{R} R\right) \subseteq{ }^{\text {ess }}{ }_{R} R$. As $R$ is left P-injective, this shows that $R$ is a left GPFring [15]. By Theorem 2.3 in [15], $\operatorname{soc}(e R)$ is simple and essential in $e R$ for every local idempotent $e$ of $R$. Write

$$
R=e_{1} R \oplus \cdots \oplus e_{n} R
$$

where $\left\{e_{1}, \cdots, e_{n}\right\}$ is a complete set of orthogonal local idempotents of $R$. It follows that $\operatorname{soc}\left(R_{R}\right) \subseteq \subseteq^{e s s} R_{R}$, so it remains to show that $R$ is right selfinjective. We do this by showing that $e_{i} R$ is injective for each $i=1, \cdots, n$.

Let $a \in E\left(e_{i} R\right)$. Then $e_{i} R+a R$ is a finitely embedded torsionless right $R$-module so, by Lemma 2.7, let $\tau: e_{i} R+a R \rightarrow \oplus_{t=1}^{k} e_{i_{l}} R$ be an embedding where each $e_{i_{t}} \in\left\{e_{1}, \cdots, e_{n}\right\}$. Then $e_{i} R+a R$ embeds in $e_{i_{j}} R$ for some $j$ by Lemma 2.6. So let $\sigma:\left(e_{i} R+a R\right) \rightarrow e_{i_{j}} R$ be monic. If $a \notin e_{i} R$ then $\sigma\left(e_{i} R\right)$ is a proper submodule of the local module $e_{i j} R$, so $\sigma\left(e_{i} R\right) \subseteq J\left(e_{i j} R\right)$ $\subseteq Z\left(R_{R}\right)$ by (2), a contradiction. Hence $a \in e_{i} R$ so $e_{i} R=E\left(e_{i} R\right)$ as required.

Recall [1] that a right artinian ring $R$ is QF if and only if $\operatorname{soc}\left({ }_{R} R\right)=\operatorname{soc}\left(R_{R}\right)$ and $\operatorname{soc}(e R)$ and $\operatorname{soc}(R e)$ are simple for every local idempotent $e$ of $R$. A result of Gomez Pardo and Guil Asensio [11] asserts that if $R$ is a right CS-ring and every cyclic right $R$ module embeds in a free module, then $R$ is right artinian. A ring $R$ is called right mininjective if each $R$-homomorphism from a simple right ideal to $R$ is given left multiplication. See [16].

Theorem 2.9. Let $R$ be a left $C S$-ring such that every cyclic right $R$-module embeds in a free module. The following conditions are equivalent.

(1) $R$ is $Q F$.

(2) $J(R) \subseteq Z\left(R_{R}\right)$.

(3) $\operatorname{soc}\left(R_{R}\right) \subseteq \operatorname{soc}\left({ }_{R} R\right)$.

(4) $R$ is right mininjective.

Proof. (1) $\Rightarrow$ (2). This is clear since $R$ is right selfinjective.

(2) $\Rightarrow(3)$. $R$ is semiperfect by Proposition 2.4 and so $\mathrm{r}(\mathrm{J})=\operatorname{soc}\left({ }_{R} R\right)$. Hence (2) gives $\operatorname{soc}\left(R_{R}\right) \subseteq r\left[Z\left(R_{R}\right)\right] \subseteq r(J)=\operatorname{soc}\left({ }_{R} R\right)$.

(3) $\Rightarrow$ (1). Observe first that $\operatorname{soc}\left(R_{R}\right) \subseteq{ }^{e s s}{ }_{R} R$ by Proposition 2.4 , $\operatorname{so} \operatorname{soc}\left({ }_{R} R\right) \subseteq \operatorname{soc}\left(R_{R}\right)$. Hence $\operatorname{soc}\left({ }_{R} R\right)=\operatorname{soc}\left(R_{R}\right)$ by (3). Next $R$ is semiperfect by Proposition 2.4, so write

$$
R=R e_{1} \oplus \cdots \oplus R e_{n}
$$

where $\left\{e_{1}, \cdots, e_{n}\right\}$ is a complete set of local orthogonal idempotents. Since $R$ is a left CS-ring with $\operatorname{soc}\left({ }_{R} R\right) \subseteq{ }^{e s s}{ }_{R} R$, each $R e_{i}$ is uniform and so $\operatorname{soc}\left(R e_{i}\right)$ is simple (and essential in $R e_{i}$ ) for each $i$. 
On the other hand, $R$ is left P-injective because $r l(T)=T$ for all right ideals $T$ of $R$, so $R e_{i} ¥ R e_{j}$ implies that $\operatorname{soc}\left(R e_{i}\right) ¥ \operatorname{soc}\left(R e_{j}\right)$ by Lemma 3.4 in [16]. It follows that $R$ is left Kasch. Hence

$$
\operatorname{soc}\left(e_{i} R\right)=e_{i} \operatorname{soc}\left(R_{R}\right)=e_{i} \operatorname{soc}\left({ }_{R} R\right)=e_{i} r(J) \cong\left(R e_{i} / J e_{i}\right)^{*} \neq 0
$$

where $M^{*}$ denotes the dual module. But $R$ is right continuous by Theorem 1.7 in [21] because $r(T)=T$ for all right ideals $T$ of $R$. Hence $\operatorname{soc}\left(e_{i} R\right)$ is simple and essential in $e_{i} R$ for each $i$ because $e_{i} R$ is uniform. Moreover, this shows that $R_{R}$ is finitely embedded. Thus every principal right $R$-module is finitely embedded (it is embedded in $R^{(n)}$ for some $n$ ) so $R$ is right artinian and hence QF.

(4) $\Rightarrow$ (3). This follows from Theorem 1.14 in [16].

$(3) \Rightarrow(4)$. This is clear because (3) $\Rightarrow(1)$.

3. Johns rings. According to Menal and Faith [8] a ring $R$ is called right Johns if $R$ is a right noetherian ring in which every right ideal is an annihilator. In [9] $R$ is called strongly right Johns if every $n \times n$ matrix ring $M_{n}(R)$ is right Johns. Strongly right Johns rings were characterized by Faith and Menal (see [9], Theorem 1.1) as the right noetherian rings that are left FP-injective (that is $R$-homomorphisms from a finitely generated submodule of a free left $R$-module $F$ into $R$ can be extended to $F$ ). It is not known if strongly right Johns rings are QF.

Several properties of right Johns rings were highlighted in [8] and a number of necessary and sufficient conditions for a strongly right Johns ring to be QF were collected in [9]. In the next proposition we deduce several new properties of these rings.

Proposition 3.1. (1) If $R$ is a right Johns ring then $\operatorname{soc}\left({ }_{R} R\right)=\operatorname{soc}\left(R_{R}\right) \subseteq{ }^{\text {ess }}{ }_{R} R$.

(2) If $R$ is strongly right Johns then the following properties hold.

(a) $\operatorname{lr}(R k)=R k$ for all minimal left ideals $R k$ of $R$.

(b) $R$ is right mininjective.

(c) $R k$ is a minimal left ideal of $R$ if and only if $k R$ is a minimal right ideal of $R$.

(d) The dual of every simple right $R$-module is simple. In particular $l(T)$ is simple for every maximal right ideal $T$ of $R$.

Proof. (1). By Lemma 2.2 in [8], it suffices to show that $l(J) \subseteq{ }^{\text {ess }} R$ where we write $J=J(R)$. If $R b \cap l(J)=0$ for some $b \in R$, then $R=r[R b \cap l(J)]=r(b)+J$ by Lemma 2.1 . Hence $r(b)=R$, so $b=0$ as required.

(2a). $R$ is left mininjective because it is left FP-injective. Hence, if $R k$ is a minimal left ideal of $R$ then $k R$ is a minimal right ideal of $R$ by [16, Theorem 1.14]. This means $J \subseteq r(k)$ so $\operatorname{lr}(k) \subseteq l(J)=S$ by Lemma 2.2 in [8], where we write $S=\operatorname{soc}\left({ }_{R} R\right)=\operatorname{soc}\left(R_{R}\right)$. Thus $\operatorname{lr}(k)$ is a semisimple left $R$-module containing $R k$, so it suffices to show that $R k \subseteq \subseteq^{\text {sss }} \operatorname{lr}(k)$. Suppose that $0 \neq y \in \operatorname{lr}(k)$. Observe first that $r(k) \subseteq r(y) \neq R$, whence $r(k)=r(y)$ and $\operatorname{lr}(k)=\operatorname{lr}(y)$. Now suppose to the contrary that $R k \cap R y=0$. Then $R=r(R k \cap R y)=r(k)+r(y)$ because $R$ is left FP-injective (see [7], Proposition 23.21). This implies that $0=l[r(k)+r(y)]$ $=\operatorname{lr}(k) \cap \operatorname{lr}(y)=\operatorname{lr}(k)$, a contradiction.

(2b). We have $r l(T)=T$ for all right ideals $T$; in particular this holds for minimal right ideals. This with (2a) proves (2b) by [16, Corollary 2.6], because $\operatorname{soc}\left({ }_{R} R\right)=\operatorname{soc}\left(R_{R}\right)$ (see [8], Lemma 2.2). 
(2c). This follows from Theorem 1.14 in [16].

(2d). $R$ is right mininjective by (2b) and it is right Kasch because $r l(T)=T$ for all right ideals $T$. Hence the dual of every simple right $R$-module is simple by Proposition 2.2 in [16]. The last statement now follows because $l(T) \cong(R / T)^{d}$.

It was shown by Faith and Menal [8] that a right Johns, left finite dimensional ring $R$ is right artinian. In fact we can say more: By Lemma 6 in [4] and Lemma 2.2 in [8], $R$ is also left artinian. Moreover, by Theorem 1.7 in [21], $R$ is right continuous. On the other hand, the example provided by Faith and Menal in [8] shows that right Johns rings need not be left or right continuous. Indeed, Example 3.8 below provides a left and right artinian, left Johns, left continuous ring which is not right continuous. However, adding a left CS-condition to a right Johns ring forces it to be quasi-Frobenius.

THEOREM 3.2. The following conditions on a ring $R$ are equivalent.

(1) $R$ is quasi-Frobenius.

(2) $R$ is a right Johns, left CS-ring.

Proof. (1) $\Rightarrow(2)$ is well known. Assume (2); we show that $\operatorname{soc}\left({ }_{R} R\right)=\operatorname{soc}\left(R_{R}\right)$ and that $\operatorname{soc}(R e)$ and $\operatorname{soc}(e R)$ are both simple for all primitive idempotents $e \in R$. Since $R$ is semiperfect by Lemma 2.2 , let $R=R e_{1} \oplus \cdots \oplus R e_{n}$ where $\left\{e_{1}, \cdots, e_{n}\right\}$ is a complete set of primitive orthogonal idempotents of $R$. Since $R$ is a left CS-ring, each $R e_{i}$ is uniform. Hence $R$ is left finite dimensional so, by the remark preceding this theorem, $R$ is left and right artinian. Moreover, Lemma 2.2 in [8] shows that $\operatorname{soc}\left({ }_{R} R\right)=\operatorname{soc}\left(R_{R}\right)$.

Since $R$ is a left CS-ring, $\operatorname{soc}(R e)$ is simple and essential in $R e$ for every local idempotent $e$ of $R$. If $0 \neq k \in \operatorname{soc}(e R)$, then $l(k) \supseteq R(1-e)+J$, a maximal left ideal of $R$. Thus $l(k)=R(1-e)+J$, so

$$
k R=r l(k)=e R \cap r(J)=e R \cap \operatorname{soc}\left(R_{R}\right)=\operatorname{soc}(e R)
$$

Thus $\operatorname{soc}(e R)$ is simple, completing the proof.

The right Johns rings are the right noetherian rings in which every right ideal is an annihilator. We now consider the artinian case.

Recall that a ring $R$ is called a right CEP-ring if every cyclic right $R$-module is essentially embedded in a projective module. These rings are right artinian [11] and right continuous [21]. If $M$ and $N$ are right $R$-modules we say (see [13]) that $M$ is weakly $N$-injective if, for every monomorphism $\sigma: N / K \rightarrow E(M)$, there exists $X_{R} \subseteq E(M)$ such that $X \cong M$ and $\sigma(N / K) \subseteq X$. It was shown by Jain and López-Permouth [13] that $R$ is a semiperfect right CEP-ring if and only if $R$ is right artinian and every indecomposable projective right $R$ module is weakly $R$-injective.

Proposition 3.3. The following conditions are equivalent for a ring $R$.

(1) $R$ is right artinian and $r l(T)=T$ for all right ideals $T$ of $R$.

(2) $R$ is right artinian and $r l(T)=T$ for all small right ideals $T$ of $R$.

(3) $R$ is right continuous and every cyclic right $R$-module embeds in a free module.

(4) $R$ is right artinian and every indecomposable projective right $R$-module is weakly $R$-injective. 
(5) $R$ is a right CEP-ring.

(6) $R$ is right perfect and every cyclic right $R$-module embeds in a free module.

(7) $R$ is semiperfect with essential left socle and every cyclic right $R$-module embeds in a free module.

Proof. (1) $\Rightarrow$ (2). This is clear.

$(2) \Rightarrow(3)$. By Lemma 1.5 in [21], we have $\operatorname{lr}(T)=T$ for all right ideals $T$ of $R$. Hence $R$ is right continuous by Theorem 1.7 in [21]. If $M=R / T$ is a cyclic right $R$-module then $M$ is torsionless because $r l(T)=T$, and $M$ is finitely embedded because $R$ is right artinian. Hence $M$ embeds in a free module by Lemma 2.7 .

(3) $\Rightarrow(4) . R$ is right artinian by Corollary 2.9 in [11], so let $\left\{e_{1}, \cdots, e_{n}\right\}$ be a basic set of local idempotents. If $1 \leq i \leq n$, we must show that $P=e_{i} R$ is weakly $R$-injective. Hence let $T$ be a right ideal of $R$, and let $\sigma: R / T \rightarrow E(P)$ be an embedding. Since $R$ is right CS, $\operatorname{soc}(P)$ is simple and essential in $P$, and so $P$ is uniform. It follows that $R / T$ is uniform. On the other hand, there is (by hypothesis) an embedding $R / T \hookrightarrow \oplus_{i=1}^{m} e_{t_{i}} R$ where $1 \leq t_{i} \leq n$ for each $i$. By Lemma 2.6 there is an embedding $\phi: R / T \rightarrow e_{t_{k}} R$ for some $t_{k} \in\{1, \cdots, n\}$, and we have

$$
\operatorname{soc}\left(e_{t_{k}} R\right)=\operatorname{soc}(\phi(R / T)) \cong \operatorname{soc}(R / T) \cong \operatorname{soc}(E(P))=\operatorname{soc}(P)
$$

It follows from Theorem 3.16 in [16] that $e_{t_{k}} R \cong P$. Now consider the following diagram.

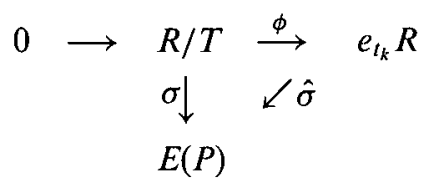

There exists $\hat{\sigma}: e_{t_{k}} R \rightarrow E(P)$ such that $\hat{\sigma} \circ \phi=\sigma$. Then $\hat{\sigma}$ is an embedding because $\phi$ is an essential embedding, and $\sigma(R / T)=\hat{\sigma}(\phi(R / T)) \subseteq \hat{\sigma}\left(e_{t_{k}} R\right) \subseteq E(P)$. So if we take $X=\hat{\sigma}\left(e_{t_{k}} R\right)$, we have $\sigma(R / T) \subseteq X \subseteq E(P)$ and $X=\hat{\sigma}\left(e_{t_{k}} R\right) \cong e_{t_{k}} R \cong P$. This proves that $P$ is weakly $R$ injective.

(4) $\Rightarrow(5)$. This follows from Theorem 5.2 in [13].

$(5) \Rightarrow(6)$. This is because right CEP-rings are right artinian by [11].

$(6) \Rightarrow(7)$. This is clear.

(7) $\Rightarrow(1)$. We have $r l(T)=T$ for all right ideals $T$ of $R$ because $R / T$ embeds in a free module. Hence $R$ is left P-injective so, by Theorem 2.3 in [15], $R$ has a finitely generated essential right socle. Since every cyclic right $R$-module is embedded in a free module, $R$ is right artinian. This proves (1).

Strengthening condition (3) in Proposition 3.3 leads to a new characterization of quasiFrobenius rings.

THEOREM 3.4. The following conditions are equivalent for a ring $R$.

(1) $R$ is quasi-Frobenius.

(2) $R$ is right $C S$ and every 2-generated right $R$-module embeds in a free module.

(3) Every 2-generated right $R$-module is essentially embedded in a projective module. 
(4) $R$ is right perfect and every 2-generated right $R$-module is embedded in a free module.

(5) $R$ is semiperfect with essential left socle and every 2-generated right $R$-module is embedded in a free module.

Proof. It is well known that (1) implies each of the other statements, (4) $\Rightarrow(5)$ is clear, and $(3) \Rightarrow(2)$ by Proposition 1.10 in [21].

$(2) \Rightarrow(5)$. By [11], $R$ is right artinian, and hence semiperfect with essential left socle.

$(5) \Rightarrow(1) . R$ is right artinian and right continuous by Proposition 3.3. In particular, $R$ is semiregular with $J=Z\left(R_{R}\right)$ by Utumi [20]. Hence $R$ is QF by Proposition 1.24 in [21].

Statement (5) in Theorem 3.4 extends a result of Rutter [19] in two directions: it replaces "right perfect" by "semiperfect with essential left socle", and it replaces "every finitely generated right $R$-module is embedded in a free module" by "every 2 -generated right $R$-module is embedded in a free module".

If we replace right artinian by left artinian in Proposition 3.3, we get the following result:

Proposition 3.5. Suppose $R$ is left artinian and $r l(T)=T$ for all finitely generated, small right ideals $T$ of $R$. Then $R$ is a right artinian, right continuous ring in which every right ideal is an annihilator. In particular, every cyclic right $R$-module embeds in a free module.

Proof. Let $K$ be any small right ideal of $R$. Since $R$ is left artinian, $l(K)=l\left\{k_{1}, \cdots, k_{n}\right\}$ for a finite subset $\left\{k_{1}, \cdots, k_{n}\right\} \subseteq K$. Thus $r l(K)=r l\left\{k_{1}, \cdots, k_{n}\right\}=\sum_{i=1}^{n} k_{i} R \subseteq K$, and so $r l(K)=K$. Hence $r l(T)=T$ for all right ideals of $R$ by Lemma 1.5 in [21], and $R$ is right continuous by Theorem 1.7 in [21]. Thus $R$ is right noetherian because it has ACC on right annihilators (it has DCC on left annihilators). Hence $R$ is right artinian by Hopkin's theorem. Finally, the last assertion follows because $r l(T)=T$ for all right ideals of $R$.

It is well known that if $R$ is a left selfinjective ring then $R$ is left continuous and satisfies the following two conditions:

(Al) $r l(T)=T$ for all finitely generated right ideal $T$ of $R$; and

(A2) $r(A \cap B)=r(A)+r(B)$ for all left ideals $A$ and $B$ of $R$.

In [21] several classes of non-injective semiperfect rings are given with annihilator conditions which guarantee the continuity of the ring. More precisely the following result was proved (see [21], Lemma 1.3 and Theorem 1.7).

Lemma 3.6. (1) Suppose $R$ is a semiperfect ring in which $\operatorname{soc}\left({ }_{R} R\right) \subseteq{ }^{e s s}{ }_{R} R, \operatorname{soc}\left(R_{R}\right) \subseteq$ ess $R_{R}$ and $\operatorname{lr}(K)=K$ for all minimal left ideals $K$ of $R$. If $L$ is a left ideal of $R$ with $L \subseteq{ }^{\text {ess }} \operatorname{lr}(L)$, then $L \subseteq{ }^{\text {ess }}$ Rf for some $f^{2}=f \in R$.

(2) Suppose $R$ is a semiperfect ring in which $\operatorname{soc}\left(R_{R}\right) \subseteq{ }^{e s s} R_{R}$ and $\operatorname{lr}(K)=K$ for all small left ideals $K$ of $R$. Then $R$ is left continuous and $\operatorname{lr}(T)=T$ for all left ideals $T$ of $R$.

It is natural to ask whether the annihilator condition in (2) of Lemma 3.6 can be replaced with condition (A2) above. Note that if $R$ satisfies (A2) then $L \subseteq{ }^{e s s} \operatorname{lr}(L)$ for all left ideals $L$ of $R$. [Indeed, if $x \in \operatorname{lr}(L)-L$ and $R x \cap L=0$, then $r(L) \subseteq r(x)$ and $r(R x \cap L)=r(x)+r(L)=R$, so $x=0$, a contradiction.] In particular every complement (closed) left ideal of $R$ is an annihilator. 
The next proposition is now an immediate consequence of the above remarks, Lemma 3.6 and Lemmas 1.1 and 1.2 in [21].

Proposition 3.7. Suppose that $R$ is a semiperfect ring in which $\operatorname{soc}\left({ }_{R} R\right) \subseteq{ }^{e s s}{ }_{R} R$, $\operatorname{soc}\left(R_{R}\right) \subseteq{ }^{e s s} R_{R}$, and $l r(K)=K$ for all minimal left ideals $K$ of $R$. Then $R$ is left continuous if either of the following conditions hold:

(1) Every complement left ideal of $R$ is an annihilator.

(2) $r(A \cap B)=r(A)+r(B)$ for all left ideals $A$ and $B$ of $R$.

EXAMPLE 3.8. Let $K$ be a field and $\sigma$ an isomorphism of $K$ onto a subfield $L$ where $[K: L]=n>1$. Let $K[X ; \sigma]$ denote the ring of twisted left polynomials over $K$. Thus $K[X ; \sigma]$ is the set of all formal polynomials in the indeterminate $X$ with coefficients from $K$ written on the left and with multiplication defined by $X a=\sigma(a) X$ for all $a \in K$. Let $R=K[X ; \sigma] /\left(X^{2}\right)$ and write $x$ for the coset determined by $X$. It can easily be verified that the only left ideals of $R$ are $0, J(R)=R x=K x$ and $R$. Thus $R$ is a two-sided artinian left continuous ring which is not right continuous. The ring $R$ satisfies the following annihilator conditions: $\operatorname{lr}(A)=A$ and $r(A \cap B)=r(A)+r(B)$ for all left ideals $A$ and $B$ of $R$. However $R$ is not right continuous and hence does not satisfy the annihilator condition $l(T \cap S)=l(T)+l(S)$ for all right ideals $T$ and $S$ of $R$.

ACKNOWLEDGEMENT. This research was carried out during a visit by both authors to the Centre de Recerca Matemàtica, Barcelona, Spain, and the authors would like to gratefully acknowledge the financial support of the CRM and the Spanish Ministerio de Education y Ciencia. The work of the first author was also supported by NSERC Grant A8075, and the work of the second author was undertaken while on a sabbatical leave from the Ohio State University during the academic year 1995-1996.

Note Added in PRoOf. Proposition 3.1 can be used to prove the following theorem which settles an open question of Faith and Menal.

THEOREM. Every strongly right Johns ring is quasi-Frobenius.

Proof. Let $R$ be a strongly right Johns ring. Then $S_{r}$ is an essential right ideal of $R$ and $l\left(S_{r}\right)=J$ by Faith and Menal [8, Lemma 2.2]. Moreover it suffices to show that $R$ is semilocal by [9, Corollary 1.3]. Since $R$ is right noetherian, we have $S_{r}=k_{1} R \oplus \ldots \oplus k_{n} R$, where each $k_{i} R$ is simple. Hence

$$
J=l\left(S_{r}\right)=l\left(k_{1} R+\oplus+k_{n} R\right)=\cap_{i=1}^{n} l\left(k_{i}\right)
$$

The result now follows from Proposition 3.1.

\section{REFERENCES}

1. F. W. Anderson and K. R. Fuller, Rings and categories of modules (Springer-Verlag, 1991).

2. J.-E. Björk, Rings satisfying certain chain conditions, J. Reine Angew. Math. 245 (1970), 63-73. 
3. V. Camillo, Commutative rings whose principal ideals are annihilators, Portugal. Math. 46 (1989), 33-37.

4. V. Camillo and M. F. Yousif, Continuous rings with ACC on annihilators, Canad. Math. Bull. 34 (1991), 462-464.

5. F. Dischinger and W. Müller, Left PF is not right PF, Comm., Alg. 14 (1986), 1223-1227.

6. C. Faith, Embedding modules in projectives. A report on a problem, Lecture Notes in Math. 951, 21-40. (Springer-Verlag, 1982).

7. C. Faith, Algebra II, Ring theory (Springer-Verlag, 1976).

8. C. Faith and P. Menal, A counter-example to a conjecture of Johns, Proc. Amer. Math. Soc. 116 (1992), 21-26.

9. C. Faith and P. Menal, The structure of Johns Rings, Proc. Amer. Math. Soc. 120 (1994), 1071-1081.

10. J. L. Gomez Pardo and P. A. Guil Asensio, Essential embedding of cyclic modules in projectives, Trans. Amer. Math. Soc. 349 (1997), 4343-4353.

11. J. L. Gomez Pardo and P. A. Guil Asensio, Rings with finite essential socle, Proc. Amer. Math. Soc. 125 (1997), 971-977. 266.

12. C. R. Hajarnavis and N. C. Norton, On dual rings and their modules, J. Algebra 93 (1985), 253-

13. S. K. Jain and S. R. López-Permouth, Rings whose cyclics are essentially embeddable in projective modules, J. Algebra 128 (1990), 257-269.

14. F. Kasch, Modules and rings (London Math. Soc. Monographs Vol 17, Academic Press, New York, 1982).

15. W. K. Nicholson and M. F. Yousif, Principally injective rings, J. Algebra 174 (1995), 77-93.

16. W. K. Nicholson and M. F. Yousif, Mininjective rings, J. Algebra 187 (1997), 548-578.

17. B. L. Osofsky, A generalization of quasi-Frobenius rings, J. Algebra 4 (1966), 373-387.

18. J. Rada and M. Saorin, On semiregular rings whose finitely generated modules embed in free, Canad. Math Bull. 40 (1997), 221-230.

19. E. A. Rutter, Two characterizations of quasi-Frobenius rings, Pacific J. Math. 30 (1969), 777-784.

20. Y. Utumi, On continuous and self-injective rings, Trans. Amer. Math. Soc. 118 (1965), 158-173.

21. M. F. Yousif, On continuous rings, J. Algebra 191 (1997), 495-509.

W. K. Nicholson

Department of Mathematics

UNIVERSity of CALGaRY

Calgary

CANADA

T2N IN4
M. F. Yousif

Department of Mathematics

Ohio State University

LiMA

OHIO 45804

USA 